\title{
Using machine learning analysis to interpret the relationship between music emotion and lyric features
}

\author{
Liang Xu ${ }^{1}$, Zaoyi Sun ${ }^{2}$, Xin Wen $^{1}$, Zhengxi Huang ${ }^{1}$, Chi-ju Chao ${ }^{3}$, Liuchang Xu ${ }^{\text {Corresp. } 4,5}$ \\ 1 Department of Psychology and Behavioral Sciences, Zhejiang University, Hangzhou, China \\ 2 College of Education, Zhejiang University of Technology, Hangzhou, China \\ 3 Department of Information Art and Design, Tsinghua University, Beijing, China \\ ${ }^{4}$ Zhejiang Provincial Key Laboratory of Forestry Intelligent Monitoring and Information Technology, Zhejiang A\&F University, Hangzhou, China \\ ${ }^{5}$ College of Mathematics and Computer Science, Zhejiang A\&F University, Hangzhou, China \\ Corresponding Author: Liuchang Xu \\ Email address: xuliuchang@zafu.edu.cn
}

Melody and lyrics, reflecting two unique human cognitive abilities, are usually combined in music to convey emotions. Although psychologists and computer scientists have made considerable progress in revealing the association between musical structure and the perceived emotions of music, the features of lyrics are relatively less discussed. Using linguistic inquiry and word count (LIWC) technology to extract lyric features in 2372 Chinese songs, this study investigated the effects of LIWC-based lyric features on the perceived arousal and valence of music. First, correlation analysis shows that, for example, the perceived arousal of music was positively correlated with the total number of lyric words and the mean number of words per sentence and was negatively correlated with the proportion of words related to the past and insight. The perceived valence of music was negatively correlated with the proportion of negative emotion words. Second, we used audio and lyric features as inputs to construct music emotion recognition (MER) models. The performance of random forest regressions reveals that, for the recognition models of perceived valence, adding lyric features can significantly improve the prediction effect of the model using audio features only; for the recognition models of perceived arousal, lyric features are almost useless. Finally, by calculating the feature importance to interpret the MER models, we observed that the audio features played a decisive role in the recognition models of both perceived arousal and perceived valence. Unlike the uselessness of the lyric features in the arousal recognition model, several lyric features, such as the usage frequency of words related to sadness, positive emotions, and tentativeness, played important roles in the valence recognition model. 


\section{Using Machine Learning Analysis to Interpret the} 2 Relationship between Music Emotion and Lyric 3 Features

4

5

Liang Xu${ }^{1}$, Zaoyi Sun ${ }^{2}$, Xin Wen ${ }^{1}$, Zhengxi Huang ${ }^{1}$, Chi-ju Chao ${ }^{3}$, Liuchang $\mathrm{Xu}^{4,5}$

${ }^{1}$ Department of Psychology and Behavioral Sciences, Zhejiang University, Hangzhou, China

${ }^{2}$ College of Education, Zhejiang University of Technology, Hangzhou, China

${ }^{3}$ Department of Information Art and Design, Tsinghua University, Beijing, China

${ }^{4}$ Zhejiang Provincial Key Laboratory of Forestry Intelligent Monitoring and Information Technology, Zhejiang A\&F University, Hangzhou, China

${ }^{5}$ College of Mathematics and Computer Science, Zhejiang A\&F University, Hangzhou, China.

Corresponding Author:

Liuchang $\mathrm{Xu}^{1}$

${ }^{1}$ No. 666, Wusu Rd., Lin'an District, Hangzhou, Zhejiang, 311300, China.

Email address: xuliuchang@zafu.edu.cn

\section{Abstract}

Melody and lyrics, reflecting two unique human cognitive abilities, are usually combined in music to convey emotions. Although psychologists and computer scientists have made considerable progress in revealing the association between musical structure and the perceived emotions of music, the features of lyrics are relatively less discussed. Using linguistic inquiry and word count (LIWC) technology to extract lyric features in 2372 Chinese songs, this study investigated the effects of LIWC-based lyric features on the perceived arousal and valence of music. First, correlation analysis shows that, for example, the perceived arousal of music was positively correlated with the total number of lyric words and the mean number of words per sentence and was negatively correlated with the proportion of words related to the past and insight. The perceived valence of music was negatively correlated with the proportion of negative emotion words. Second, we used audio and lyric features as inputs to construct music emotion recognition (MER) models. The performance of random forest regressions reveals that, for the recognition models of perceived valence, adding lyric features can significantly improve the prediction effect of the model using audio features only; for the recognition models of perceived arousal, lyric features are almost useless. Finally, by calculating the feature importance to interpret the MER models, we observed that the audio features played a decisive role in the recognition models of both perceived arousal and perceived valence. Unlike the uselessness of the lyric features in the arousal recognition model, several lyric features, such as the usage 
39 frequency of words related to sadness, positive emotions, and tentativeness, played important

40 roles in the valence recognition model.

41

\section{Introduction}

43 The pursuit of emotional experience is a vital motivation for listening to music (Juslin \&

44 Sloboda, 2001; Juslin \& Laukka, 2004), and the ability to convey emotions ensures the important

45 role of music in human life (Yang, Dong, \& Li, 2018). Therefore, the relationship between music

46 and perceived emotional expression has attracted increasing academic attention in recent decades

47 (Swaminathan \& Schellenberg, 2015). Most of the related studies have focused on investigating

48 the association between musical structure and perceived emotions. For example, psychologists

49 have made considerable progress in revealing structural factors (e.g., tempo, pitch, and timbre),

50 indicating different emotional expressions (Gabrielsson, 2016), and computer scientists have

51 focused on extracting features from audio (audio most commonly refers to sound, as it is

52 transmitted in signal form; e.g., Mel-frequency cepstrum coefficients and Daubechies wavelet

53 coefficient histograms) to automatically identify music emotion (Yang, Dong, \& Li, 2018).

54 Previous works have shown that sound features were highly correlated with music emotions

55 (Gabrielsson, 2016; Yang, Dong, \& Li, 2018), but the lyric features have been relatively less

56 discussed. Besson et al. (1998) proved that melodic and lyrical components in music are

57 processed independently. Although melodic information may be more dominant than lyrics in

58 conveying emotions (Ali \& Peynircioğlu, 2006), investigating the relationship between lyrical

59 structure and the perceived emotion of music in detail is still necessary.

60

61 Music emotion studies related to lyrics have often focused on investigating the differences

62 between the presence and absence of lyrics (Ali \& Peynircioğlu, 2006; Brattico et al., 2011; Yuet

63 al., 2019), the effects of consistency or differences in melodic and lyrical information (Morton \&

64 Trehub, 2001; Vidas et al., 2019), or the effects of lyrics with different meanings (Batcho et al.,

65 2008; Stratton \& Zalanowski, 1994). While lyric structures and features have been rarely studied,

66 melodic information has been processed in previous psychology studies (Gabrielsson, 2016;

67 Swaminathan \& Schellenberg, 2015; Xu, Wen et al., 2020). On the other hand, with the

68 development of natural language processing (NLP) technology, different lyric features have been

69 widely extracted and analyzed in music emotion recognition (MER) studies (e.g., Malheiro et al.,

70 2016; Delbouys et al., 2018), a field investigating computational models for detecting music

71 emotion (Aljanaki, Yang, \& Soleymani, 2017; Chen, Lee et al., 2015). These MER studies have

72 typically focused on improving the prediction effect of constructed models but have not

73 interpreted the model and variables. Thus, can the structural factors of lyrics be analyzed in more

74 detail by combining NLP technology? If so, this may facilitate the understanding of the

75 relationship between lyrics and perceived emotions. Therefore, the present study investigated the

76 effects of various lyric features on the perceived emotions in music.

77

78

Related work 


\section{Emotion Perception in Music with Lyrics}

80 Melody and lyrics, reflecting two unique human cognitive abilities, are usually combined in 81 music to convey various information (Gordon et al., 2010). A melody is a linear succession of 82 musical tones that the listener perceives as a single entity (van Waesberghe, 1955); and lyrics is 83 the composition in verse which is sung to a melody to constitute a song. Besson et al. (1998) and 84 Bonnel et al. (2001) have shown that the melodic and lyrical components are processed

85

86

87

88

89

90

91

92

93

94

95

96

97

98

99

100

101

102

103

104

105

106

107

108

109

110

111

112

113

114

115

116

117

118 independently. However, they are often integrated in such a way that melodic information is enhanced by the simultaneous presence of lyrics (Serafine, Crowder, \& Repp, 1984; Serafine et al., 1986). Studies have been continuously updated to investigate the interaction or independence of melody and lyrics by using melody only (Bonnel et al., 2001; Brattico te al., 2011; Kolinsky et al., 2009), lyrics only (Fedorenko et al., 2009; Poulin-Charronnat et al., 2005; Yu, Wu et al., 2019), or both (Gordon et al., 2010; van Besouw, Howard, \& Ternström, 2005). The role of melody and lyrics in music emotion perception is a vital research focus.

As the soul of music, perceived emotion has been widely discussed in recent decades (Swaminathan \& Schellenberg, 2015). Ali and Peynircioğlu (2006) investigated differences in melodies and lyrics conveying the same and mismatched emotions and confirmed the dominance of melody in music emotional information processing. Additionally, they observed that lyrics can strengthen the perception of negative emotions but weaken the perceived positive emotions. A computational study (Hu, Downie, \& Ehmann, 2009) found that negative emotion classification accuracy was improved by adding lyric information, while the opposite effect was obtained for the classification of positive emotions. In contrast, the results of Laurier, Grivolla, and Herrera (2008) showed that lyrics can facilitate the recognition of happy and sad musical emotions but not angry and violent emotions. Explanatory studies were also conducted instantly following the observed phenomena. Pieschl and Fegers (2016) advocated using short-term effects on cognition and affect to explain the power of lyrics. By comparing music with and without lyrics, evidence from functional magnetic resonance imaging also indicated the importance of lyrics for negative musical emotions (Brattico et al., 2011). Following the work of Brattico et al. (2011), neural mechanisms have been continually studied in recent years (e.g., Greer et al., 2019; Proverbio, De Benedetto, \& Guazzone, 2020). In sum, although subtle conflicts exist in different studies, the substantial role of lyrics in music emotion perception is consistent.

The aforementioned theoretical findings have also been supplemented or utilized in other fields. For instance, developmental psychology studies have proven that lyrical information dominates children's judgment of music emotion (Morton \& Trehub, 2001; Morton \& Trehub, 2007), but adults rely on melody (Ali \& Peynircioğlu, 2006; Vidas et al., 2019); music therapy studies have widely conducted lyric analyses to extend the understanding of clients' emotional and health states (Baker et al., 2009; O'Callaghan \& Grocke, 2009; Silverman, 2020; Viega \& Baker, 2016); and in computational studies, lyrical information used as additional inputs can significantly improve the predictive effects of MER models (Laurier, Grivolla, \& Herrera, 2008; 
119 Malheiro et al., 2018; Yu, Tang et al., 2019). These studies provide a development direction and

120

121

122

123

124

125

126

127

128

129

130

131

132

133

134

135

136

137

138

139

140

141

142

143

144

145

146

147

148

149

150

151

152

153

154

155

156

157

158

practical value of basic lyrics research and encourage the optimization of basic research.

One of the limitations in previous behavioral research is that the lyric was always treated as a complete object. Studies usually investigated the differences between the presence and absence of lyrics (Ali \& Peynircioğlu, 2006; Yu, Wu et al, 2019) or the effects of lyrics with a certain meaning (e.g., lyrics expressing homesickness in Batcho et al., 2008, or happy and sad lyrics in Brattico et al., 2011) but rarely analyzed the elements extracted from lyrics. In melody-related research, various musical structural factors (e.g., mode, timbre, tempo, and pitch) have been studied (Juslin \& Laukka, 2004), and the association between perceived emotion and structural factors has been repeatedly verified in recent decades (Gabrielsson, 2016; Hunter \& Schellenberg, 2010). Therefore, to better understand the relationship between lyrics and music perception emotions, can similar methods be used to analyze the structural factors of lyrics? In addition, unlike musical structural factors, which have been summarized in musicology, analyzing lyrical factor types remains a challenge. We noticed that recent linguistic and NLPbased computational studies can provide inspiration.

\section{NLP-based Lyric Features}

NLP technology has been widely used to analyze the emotions expressed or perceived in texts, such as book reviews (Zhang, Tong, \& Bu, 2019), opinions on social media platforms (Xu, Li et al., 2020), movie reviews (Kaur \& Verma, 2017; Lu \& Wu, 2019), party statements (Haselmayer \& Jenny, 2017), and song lyrics (Rachman, Samo, \& Fatichah, 2019). Knowledge-based approaches and machine learning-based approaches are two common approaches used for emotional analysis (Liu \& Chen, 2015). The knowledge-based approach is an unsupervised approach that uses an emotional dictionary or lexicon (a list of words or expressions used to express human emotions) to label emotional words in text (Liu, 2012). Thus, a high-quality emotional dictionary is the basis of this approach. In contrast, the machine learning approach is usually a supervised approach that requires a labeled dataset to construct emotion recognition models (Peng, Cambria, \& Hussain 2017). It usually constitutes a process of (a) extracting text features (including lexical features, syntactic features and semantic features), (b) using machine learning algorithms to construct the relationship between extracted features and labeled emotions, and (c) predicting the emotions of untagged texts.

When conducting emotion analyses of song lyrics, machine learning-based approaches have been more prevalent in the past two decades. Laurier, Grivolla, and Herrera (2008) used lyric feature vectors based on latent semantic analysis (LSA) dimensional reduction and audio features to conduct music mood classification. They found that standard distance-based methods and LSA were effective for lyric feature extraction, although the performance of lyric features was inferior to that of audio features. Petrie, Pennebaker, and Sivertsen (2008) conducted linguistic inquiry and word count (LIWC) analyses to explore the emotional changes in Beatles' lyrics over time.

PeerJ Comput. Sci. reviewing PDF | (CS-2021:07:63905:1:0:NEW 29 Sep 2021) 
159 Panda et al. (2013) used support vector machines, K-nearest neighbors, and naïve Bayes

160

161

162

163

164

165

166

167

168

169

170

171

172

173

174

175

176

177

178

179

180

181

182

183

184

185

186

187

188

189

190

191

192

193

194

195

196

197

algorithms to map the relationship between music emotion and extracted audio and lyric features. In a recent study, Zhou, Chen, and Yang (2019) applied unsupervised deep neural networks to perform feature learning and found that this method performed well for audio and lyric data and could model the relationships between features and music emotions effectively. Notably, traditional MER research focuses on improving the prediction effect of the MER models, while our study attempted to use an interpretable way to investigate the relationship between lyrics features and music emotions.

Previous studies have shown a variety of methods for extracting lyric features. Although the lyric feature vectors and the deep learning-based features performed well in MER studies, the meaning of these features is often difficult to understand. Thus, considering the interpretability of lyric features, this study selected the LIWC-based method to extract lyric features. The LIWC software package was first developed to analyze text for more than 70 language dimensions by Pennebaker, Francis, and Booth (2001). It has been applied for text analysis in psychological health (Slatcher \& Pennebaker, 2006), physical health (Pennebaker, 2004), and lyric studies (Petrie, Pennebaker, \& Sivertsen, 2008; Pettijohn \& Sacco Jr, 2009). The simplified Chinese version of LIWC (SC-LIWC; Gao et al., 2013; Zhao et al., 2016), which expanded text features for more than 100 dimensions, was also developed in recent years. This technology was considered for lyric feature extraction in this study.

\section{The Present Research}

In sum, the present study investigates the association between LIWC-based lyric features and the perceived emotions of Chinese songs. First, the direct relationships between the independent variables (lyric features) and the dependent variables (perceived emotions of music) are investigated through correlation analysis. Then, a computational modeling method is considered to examine the effects of lyric features on music emotion perception. Since melody and lyrics are inseparable in music, we use the audio and lyric features extracted in music to predict the perceived emotions. By comparing the prediction effects of the models that use lyric features as input and that lack lyric features, we can intuitively witness the effect of lyrics. Moreover, using interpretable and nonlinear machine learning methods to construct prediction models, different forms of association between lyrics and perceived emotions can be observed (Vempala \& Russo, 2018; Xu, Wen et al., 2020). Finally, the constructed MER models are also of practical value because the recognized music emotion information can be used in various fields, such as music recommendation (Deng et al., 2015), music information retrieval (Downie, 2008), and music therapy (Dingle et al., 2015).

\section{Materials \& Methods}

\section{Data Collection}

Peer) Comput. Sci. reviewing PDF | (CS-2021:07:63905:1:0:NEW 29 Sep 2021) 
198

199

200

201

202

203

204

205

206

207

208

209

210

211

212

213

214

215

216

217

218

219

220

221

222

223

224

225

226

227

228

229

230

231

232

233

234

235

236

237

The original music files and emotion annotation results were obtained from the PSIC3839 dataset, a public-free dataset for MER studies (Xu, Yun et al., 2020, unpublished data). In this dataset, arousal and valence scores of 3839 songs popular in China were manually annotated by 87 university students using 5-point Likert scales. Based on the multi-dimensional emotion space model, Lang (1995) suggested that emotions can be categorized in a two-dimensional space by valence and arousal; valence ranges from negative to positive, and arousal ranges from passive (low) to active (high). In the PSIC3839 dataset, valence was evaluated from -2 (negative) to 2 (positive), and arousal was evaluated from -2 (not at all) to 2 (very much). We then downloaded the lyrics of songs from NetEase Cloud Music (https://music.163.com/), a popular music site in China. Considering that the annotators of the PSIC3839 dataset are all native Chinese, only 2372 songs with Chinese lyrics were retained for subsequent analysis.

\section{Lyric Feature Extraction}

To extract the lyric features, the raw data need to be preprocessed. First, since the raw data of lyrics were downloaded online, they contained a large amount of unwanted information, such as singer, composer, and lyricist names and the words "man" and "woman" in duet songs. Thus, we manually filtered these unwanted information elements. Second, unlike English texts that are directly composed of separated words, Chinese texts require special tools to divide them into separate words for analysis. For example, the sentence "he feels happy" in English text is "hefeelshappy" in Chinese text. Therefore, this study used the Chinese word segmentation tool in the Language Technology Platform (Che, Li, \& Liu, 2010) for text segmentation. Through the above steps, the raw lyrics of each song were processed into Chinese words arranged in order.

After the above data preprocessing, we used SC-LIWC (Gao et al., 2013; Zhao et al., 2016) to extract the lyric features. A total of 98 types of lyric features were calculated for each song, such as the total number of words (WordCount), the proportion of positive emotion words (PosEmo), and the proportion of swear words (Swear). For example, the lyric feature PosEmo, reflecting the usage frequency of positive emotion words in each song, is calculated by dividing the number of positive emotion words in SC-LIWC by the total number of words. All the extracted features are listed and introduced in Supplemental Materials Table S1

\section{Audio Feature Extraction}

For audio features, this study considered both rhythmic features (by beat and tempo detection) and spectral features related to timbre, pitch, harmony, and so forth (e.g., Mel-frequency cepstrum coefficients, MFCCs). Audio signal preprocessing was first conducted by (a) using a $22050 \mathrm{~Hz}$ sampling rate to sample each song and (b) using a short-term Fourier transform to obtain the power spectrogram. Then, using the librosa toolkit (McFee et al., 2015), a total of nine low or middle features were extracted, including MFCCs, spectral centroid, spectral bandwidth, spectral roll-off, spectral flatness, spectral contrast, tonal centroid features (tonnetz), chromagram, and tempo. Different spectral features were calculated in different ways. For 
238

239

240

241

242

243

244

245

246

247

248

249

250

251

252

253

254

255

256

257

258

259

260

261

262

263

264

265

266

267

268

269

270

271

\section{.}

1

instance, MFCCs were calculated by selecting the lower cepstral coefficients of a Mel-scaled spectrogram, which was generated by using a Mel filter bank to filter the spectrogram (Meyer \& Kollmeier, 2009).

Since the extracted features of each song were represented in a subspace of high dimensionality, we conducted feature reduction of each type of feature to reduce the storage and computational space. Principal component analysis (PCA), widely used in MER studies (Xu, Wen et al., 2020; Yang, Dong, \& Li, 2018), was applied to reduce the dimensionality of features. After conducting the PCA, we selected and combined the top 50 dimensions of each type of audio feature as model inputs. Finally, we scaled each continuous audio feature to a value of 0 to 1 via min-max scaling (Kahng, Mantik, \& Markov, 2002). The above processed features were used as the final audio inputs of the computational models.

\section{Construction of Computational Models}

The proposed modeling method is shown in Figure 1. Since the annotation results of the perceived valence and arousal values are continuous variables, this study formulated the construction of computational models as a regression problem, which predicts a real value from observed features (Sen \& Srivastava, 2012). We used the audio and lyric features as model inputs and the perceived emotion values as ground truth. To explore the effects of lyric features, three types of input sets were considered: (a) audio features only; (b) lyric features only; and (c) combining audio and lyric features. In addition, the ground truth values were also scaled to a value of 0 to 1 via min-max scaling before modeling.

Two machine learning algorithms were then considered to map the inputs and perceived emotion values (ground truth data). Multiple linear regression (MLR) was used as the baseline algorithm. Random forest regression (RFR), showing good performance in MER tasks (e.g., Xu, Wen et al., 2020; Xu et al., 2021), was used as the main algorithm. For each RFR model, we used a grid parameter search to obtain the best modeling parameters. The performances of our models were evaluated by the tenfold cross-validation technique. The prediction accuracy of each regressor was measured by $R^{2}$ statistics and the root mean-squared error (RMSE) as follows:

$$
R^{2}=1-\frac{\sum_{i=1}^{N}\left(X_{i}-Y_{i}\right)^{2}}{\sum_{i=1}^{N}\left(X_{i}-\bar{X}\right)^{2}}
$$

$$
R M S E=\sqrt{\frac{1}{N} \sum_{i=1}^{N}\left(X_{i}-Y_{i}\right)^{2}}
$$


272 where $\boldsymbol{X}_{\boldsymbol{i}}$ is the perceived emotion value (ground truth) of each song, $\overline{\boldsymbol{X}}$ is the mean value of the 273 perceived emotion values, $\boldsymbol{Y}_{\boldsymbol{i}}$ is the predicted result of each song, and $\boldsymbol{N}$ is the number of testing 274 samples in the tenfold cross-validation technique.

\section{Results}

\section{Data Distribution}

277 As the first step in exploring the data, we created a preliminary description of the emotional 278 annotation results of all songs and the content of the lyrics. Figure 2 a shows the distribution of 279 annotated emotions of songs in the valence-arousal emotion space. We observed that a large 280 proportion of the songs fell under the third quadrant (37.94\%; low arousal and negative), 281 followed by the first (34.49\%; high arousal and positive) and fourth quadrants (17.07\%; high 282 arousal and negative). Pearson correlation analysis shows that the perceived arousal values are

283

284

285

286

287

288

289

290

291

292

293

294

295

296

297

298

299

300

301

302

303

304

305

306

307

308

309

310

311 positively correlated with valence $(\mathrm{r}(2371)=0.537, p<.001)$.

We then calculated the usage frequency of different words in song sets with different emotions. Excluding commonly used personal pronouns (e.g., "I" and "you") and verbs (e.g., "is" and "are"), the top used words are presented in the word clouds (see Figure $2 b$ ). We observed that the words "love" and "world" frequently appear in every quadrant of the valence-arousal emotion space, meaning that these are two popular song themes. The words "happy" and "beautiful" frequently appear in positive songs, whereas the words "lonely" and "recall" frequently appear in negative songs. The above results allow us to intuitively see the difference in word usage in different emotional songs.

\section{Correlation Analysis between Perceived Emotions and Lyric Features}

In this part, we analyzed how well the independent variables (lyric features) accounted for the dependent variables (perceived arousal and valence values). The lyric features most relevant to arousal and valence are shown in Table 1 and Table 2, respectively. For instance, using Pearson correlation analysis, we found that the perceived arousal values were positively correlated with the total number of words in songs (WordCount, $\mathrm{r}(2371)=0.206, p<.01$ ), the mean number of words per sentence (WordPerSentence, $\mathrm{r}(2371)=0.179, p<.01$ ), the ratio of Latin words (RateLatinWord, $\mathrm{r}(2371)=0.183, p<.01)$, and the proportion of words related to achievement (Achieve, $\mathrm{r}(2371)=0.111, p<.01)$ and were negatively correlated with the proportion of words related to the past $(t$ Past, $\mathrm{r}(2371)=-0.124, p<.01)$ and the proportion of words related to insight (Insight, $\mathrm{r}(2371)=-0.122, p<.01)$. For valence, we observed that the perceived valence values were negatively correlated with the proportion of negative emotion words (NegEmo, r $(2371)=$ $0.364, p<.01)$ and proportion of words related to sadness $(\mathrm{Sad}, \mathrm{r}(2371)=-0.299, p<.01)$. The entire correlation results are presented in Supplemental Materials TabelS2. The correlation results only reveal the linear relationships between perceived emotions and lyric features. Thus, we then used machine learning methods to investigate other types of relationships.

\section{Model Prediction Results}


312 In this section, we used MLR and RFR algorithms, different input sets (audio features, lyric 313 features, and combined features), and different ground truth data (arousal and valence) to 314 construct MER models. To obtain relatively good models, a grid parameter search was first 315 conducted to obtain the best performing parameters for each RFR model (results are shown in 316 Table 3).

317

318

319

320

321

322

323

324

325

326

327

328

329

330

331

332

333

334

335

336

337

338

339

340

341

342

343

344

345

346

347

348

349

350

351

After parameter searching, the performances of the constructed models, evaluated by the tenfold cross-validation technique, are presented in Figure 3. Since the tenfold cross-validation technique, using 10\% data as the testing data and using the remaining $90 \%$ instances as training data to train regressor, used the same folds in the evaluation of different models, a tenfold paired sample $t$-test can be applied to compare the model results. For the algorithms, the RFR algorithm performed better than the MLR algorithm in all the constructed models. For example, ten-fold paired sample t-test showed that, using combined features to predict perceived arousal values, the RFR-based model reached a mean $R^{2}$ value of 0.631 and a mean RMSE value of 0.147 , significantly better than the MLR-based model $\left(R^{2}=0.532, \mathrm{t}(9)=4.206, p<.01, d=1.883\right.$; $\mathrm{RMSE}=0.165, \mathrm{t}(9)=-4.012, p<.01, d=-1.960)$. Therefore, the subsequent analysis was only conducted on the models based on the RFR algorithm.

For the recognition models of perceived arousal values, paired sample $t$-test showed that the model using audio features as inputs performed significantly better than the model using lyric features $\left(R^{2}: \mathrm{t}(9)=36.335, p<.001, d=15.219\right.$; RMSE: $\left.\mathrm{t}(9)=-34.693, p<.001, d=-12.167\right)$. Although the model using combined features $\left(R^{2}=0.631\right.$, RMSE $\left.=0.147\right)$ performed slightly better than the model using audio features $\left(R^{2}=0.629, \mathrm{RMSE}=0.147\right)$, there was no significant effect $\left(R^{2}: \mathrm{t}(9)=0.134, p=.896, d=0.063\right.$; RMSE: $\left.\mathrm{t}(9)=-0.231, p=.822, d=-0.114\right)$. These results revealed that the perceived arousal of music mainly depends on audio information, while lyrics are not important.

For valence, paired sample $t$-test showed that, although the best performing recognition model of perceived valence values performed worse than the arousal model $\left(R^{2}: \mathrm{t}(9)=-6.700, p<.001, d\right.$ $=-3.271$; RMSE: $\mathrm{t}(9)=11.539, p<.001, d=6.094)$, both lyric and audio features played important roles. The RFR-based model using audio features only reached a mean $R^{2}$ value of 0.371 and a mean RMSE value of 0.214 , and the model using lyric features as inputs reached a mean $R^{2}$ value of 0.370 and a mean RMSE value of 0.214 . When the audio and lyric features were combined as inputs, the new model achieved a mean $R^{2}$ value of 0.481 and a mean RMSE value of 0.194 , significantly better than the previous two models. These results indicated that the perceived valence of music was influenced by both audio and lyric information.

\section{Model Interpretability}

In the last step, we attempted to explain the best performing RFR-based models by examining the information gain of features. Quiroz, Geangu, \& Yong (2018) noted that models constructed 
352

353

354

355

356

357

358

359

360

361

362

363

364

365

366

367

368

369

370

371

372

373

374

375

376

377

378

379

380

381

382

383

384

385

386

387

388

389

390

using the RFR algorithm can be interpreted by calculating feature importance. Thus, the feature importance of the best performing recognition models of the perceived arousal and valence was calculated and is presented in Figure 4. Since tenfold cross-validation was used to evaluate the models, the coefficients of feature importance might differ when predicting different test sets (Xu, Wen et al., 2020). Thus, the distribution of feature importance was arranged in descending order of the mean value, and only the top 30 features were included for visibility.

In the arousal recognition model, the audio features played a decisive role, which accounted for $95.01 \%$ of the model. The first PCA components of spectral flatness, spectral contrast, chromagram, MFCCs, and spectral bandwidth are the five most contributing features, accounting for $30.71 \%, 12.38 \%, 8.77 \%, 8.62 \%$, and $4.26 \%$, respectively. For the lyric features, the feature importance results are similar to the results of the correlation analysis. The total number of words in songs (WordCount) and the mean number of words per sentence (WordPerSentence) were the top two contributing lyric features, accounting for $0.19 \%$ and $0.15 \%$ of the model.

For valence, the audio features explained $73.44 \%$ of the model. The first PCA components of spectral contrast and spectral flatness also showed good predictive effects on the perceived valence, accounting for $24.82 \%$ and $10.42 \%$ of the model, respectively. The proportion of negative emotion words (NegEmo) was the most important lyric feature (accounting for 5.32\%), followed by the proportion of words related to sadness ( $\mathrm{Sad}, 0.56 \%)$, positive emotions (PosEmo, 0.39\%), tentativeness (Tentat, $0.23 \%$ ), and so on. These findings also support the opinion that lyric features can provide more information for recognition models of valence than for recognition models of arousal.

\section{Discussion}

This study investigated the effects of LIWC-based lyric features on the perceived arousal and valence of music. We first explored the data distribution, and several interesting results were found. First, the emotional distribution (in the valence-arousal emotion space) of music in this study is similar to previous works. Various studies have shown that the perceived valence and arousal of music were positively correlated (e.g., Chen, Yang et al., 2015; Greenberg et al., 2016; Speck et al., 2011). This reveals that the relationship between valence and arousal in music is relatively constant. Second, analyzing the songs' word usage frequency in different quadrants of the valence-arousal emotion space, we then found that the word "love" frequently appears in each quadrant. The cross-cultural study of Freeman (2012) has shown that "romantic love" is the top topic category of Chinese-language pop songs ( $82.5 \%$ ), while Western pop songs with the topic of "romantic love" accounted for only $40 \%$. The high usage frequency of the word "love" in this study further confirms that love-themed songs are the mainstream in Chinese pop music. In addition, we observed that the words "happy" and "beautiful" frequently appear in positive songs, whereas the words "lonely" and "recall" frequently appear in negative songs. This 
391

392

393

394

395

396

397

398

399

400

401

402

403

404

405

406

407

408

409

410

411

412

413

414

415

416

417

418

419

420

421

422

423

424

425

426

427

428

429

430

intuitive phenomenon shows that, in general, perceived music emotions are related to lyrics, which encourages us to further explore lyric features.

Although LIWC-based lyric features have been considered in computational studies to improve the model effect (e.g., Hu, Chen, \& Yang, 2009; Malheiro et al., 2016), the role of LIWC-based lyric features has never been analyzed and discussed individually. Thus, this study then investigated the linear relationship between each lyric feature and the perceived emotion of music. In general, valence is more correlated with the features reflecting the meaning of the lyric text, while arousal is more correlated with the features reflecting the structure of the lyrics. For example, we observed that the perceived valence values were negatively correlated with the usage frequency of words related to negative emotions (e.g., "sad", "horrible", "angry", and "bitter"), tentativeness (e.g., "seem", "dim", "guess", and "dubitation"), insight (e.g., "understanding", "notice", "analyze", and "know"), and exclusiveness (e.g., "exclude", "forget", "ignore", and "cancel"). It is obvious that the usage of emotional words is related to perceived emotions because emotional words shape emotional percepts (Gendron et al., 2012). Words related to tentativeness are often used in sad love songs to express doubts about love (e.g., "In the next few days, I guess you won't show up either"), and insight words are often used with negative words to portray the sad atmosphere (e.g., "no one notices me, only a small raindrop accompanies me to wait for dawn"). We believe that some words in lyrics are often used to describe certain behaviors, feelings or scenes, which are related to negative emotions. For instance, nostalgia, characterized by sadness, insomnia, loss of appetite, pessimism, and anxiety (Batcho et al., 2008; Havlena \& Holak, 1991), is one of the themes of songs. Thus, words that describe nostalgia in lyrics are often related to negative emotions. This phenomenon may appear in songs with various themes, such as farewell, war, and tragic love stories.

For arousal, we observed that the perceived arousal values were positively correlated with the total number of lyric words (WordCount) and the mean number of words per sentence (WordPerSentence). We wonder that the size of music may play an important role, because the duration of music is positively correlated with the total number of lyric words $(r(2371)=0.219, p$ $<.01$ ); that is, the longer the music, the more words are in the lyrics. Unfortunately, as shown in Supplemental Materials Figure S1, the duration of music was negatively correlated with the arousal values $(\mathrm{r}(2371)=-0.111, \mathrm{p}<.01)$. In addition, Holbrook and Anand $(1990)$ found that the tempo of music is positively correlated with listeners' perceived arousal. Thus, another assumption is that fast-paced songs tend to match more lyric words. Unfortunately, the above hypothesis was not confirmed in the current data set $(\mathrm{r}(2371)=-0.039, p=.058)$. This result reminds us to analyze the relationship between audio features and the number of words. We found that the total number of lyric words was positively correlated with chromagram and negatively correlated with MFCCs (see Supplemental Materials Table S3). Chromagrams reflect the pitch components of music over a short time interval (Schmidt, Turnbull, \& Kim, 2010). Previous work on screams has found a significant tendency to perceive higher-pitched screams 
431 as more emotionally arousing than lower-pitched screams (Schwartz \& Gouzoules, 2019).

432 However, whether the above phenomenon holds in music is still unknown, and it is worthy of

433

434

435

436

437

438

439

440

441

442

443

444

445

446

447

448

449

450

451

452

453

454

455

456

457

458

459

460

461

462

463

464

465

466

467

468

469

470 further research. While MFCCs reflect the nonlinear frequency sensitivity of the human auditory system (Wang et al., 2012), it is difficult to map well-known music features in conventional musical writing. The low-level audio features in this study may not directly explain the relationship between melody features and lyrics. In fact, McVicar, Freeman, and De Bie (2011) found it hard to interpret the correlations between arousal and lyric features. Therefore, how to map the relationships among arousal, melody, and lyrics still needs further investigation.

The above correlation analysis reflects the direct connection between lyric features and perceived emotions. We then used audio features and lyric features to construct MER models. By comparing the results of models and calculating feature importance to interpret the constructed models, we investigated the role of lyric features and obtained two major discoveries. First, we found that, compared with lyric features, audio features played a decisive role in the MER models for both perceived arousal and perceived valence. From the perspective of computational modeling, this finding confirms previous conclusions that melodic information may be more dominant than lyrics in conveying emotions (Ali \& Peynircioğlu, 2006). However, previous works used individual moods affected by music to evaluate the ability of music to convey emotions (Ali \& Peynircioğlu, 2006; Sousou, 1997), which is not equivalent to the perceived emotions of music. Thus, our study provided more direct evidence that melody information plays a decisive role in the perception of music emotions, and we believe that this result can be generalized to all countries. The second major finding was that, unlike the uselessness of the lyric features in the arousal recognition model, lyric features can significantly improve the prediction effect of the valence recognition model. Feature importance analysis also shows that lyric features, such as the proportion of words related to sadness ( $\mathrm{Sad})$, positive emotions (PosEmo), and tentativeness (Tentat), played important roles in the valence recognition model. This finding was consistent with that of Hu, Downie, \& Ehmann (2009), which showed that lyrics can express the valence dimension of emotion but usually do not express much about the arousal dimension of emotion, rather than the opposite finding shown by Malheiro et al. (2016). We hypothesize that the main reason for the difference in results is that our study and the study of Hu, Downie, \& Ehmann (2009) both focused on Chinese music and participants, but the study of Malheiro et al. (2016) was conducted in Portugal. Cross-cultural studies have shown that although listeners are similarly sensitive to musically expressed emotion (which is facilitated by psychophysical cues; Argstatter, 2016; Balkwill \& Thompson, 1999), differences still exist (Zacharopoulou \& Kyriakidou, 2009). Therefore, we believe that in the Chinese environment, perceived music valence is affected by lyrics, although its influence is not as strong as that of melody information.

As mentioned before, this study is also of practical value. The computational modeling method was first proposed in the field of MER, which aims to automatically recognize the perceptual 
471 emotion of music (Yang, Dong, \& Li, 2018). There are many existing songs, but it is difficult for 472 people to manually annotate all emotional information. Thus, MER technology is urgently 473 needed and has made great progress in the past two decades. Recognized emotion information 474 can be used in various application scenarios (Deng et al., 2015; Dingle et al., 2015; Downie, 475 2008). The collected data and proposed methods in this study can also provide references for 476 future MER research. Notably, the computational modeling methods in MER studies pursue 477 model effects and prediction accuracy, but when they are applied in music psychology research, 478 the interpretability of the model should be taken into account (Vempala \& Russo, 2018; Xu, Wen 479 et al., 2020). Therefore, we chose MLR and RFR to construct MER models. How to integrate 480 machine learning methods into music psychology research more effectively still needs more 481 exploration.

482

483

\section{Conclusions}

484

485

486

487

488

489

490

491

492

493

494

495

496

497

498

499

500

501

502

503

504

505

506

507

508

509

The present work investigated the effects of LIWC-based lyric features on the perceived arousal and valence of music by analyzing 2372 Chinese songs. Correlation analysis shows that, for example, the perceived arousal of music was positively correlated with the total number of lyric words $($ WordCount, $\mathrm{r}(2371)=0.206, p<.01)$ and the mean number of words per sentence (WordPerSentence, $\mathrm{r}(2371)=0.179, p<.01)$ and was negatively correlated with the proportion of words related to the past $($ tPast $, \mathrm{r}(2371)=-0.124, p<.01)$ and insight $($ Insight, $\mathrm{r}(2371)=-0.122$, $p<.01)$. The perceived valence of music was negatively correlated with the proportion of negative emotion words $(\mathrm{NegEmo}, \mathrm{r}(2371)=-0.364, p<.01)$ and the proportion of words related to sadness $(\operatorname{Sad}, \mathrm{r}(2371)=-0.299, p<.01)$. We then used audio and lyric features as inputs to construct MER models. The performance of RFR-based models shows that, for the recognition models of perceived valence, adding lyric features can significantly improve the prediction effect of the model using audio features only; for the recognition models of perceived arousal, lyric features are almost useless. Calculating the importance of features to interpret the MER models, we observed that the audio features played a decisive role in the recognition models of both perceived arousal and perceived valence. Unlike the uselessness of the lyric features in the arousal recognition model, several lyric features, such as the proportion of words related to sadness $(S a d)$, positive emotions (PosEmo), and tentativeness (Tentat), played important roles in the valence recognition model.

\section{References}

Ali SO, Peynircioğlu ZF. 2006. Songs and emotions: are lyrics and melodies equal partners? Psychology of Music 34:511-534 DOI: 10.1177/0305735606067168.

Aljanaki A, Yang YH, Soleymani M. 2017. Developing a benchmark for emotional analysis of music. PLoS ONE 12:e0173392 DOI: 10.1371/journal.pone.0173392.

Argstatter H. 2016. Perception of basic emotions in music: Culture-specific or multicultural? Psychology of Music 44:674-690 DOI:10.1177/0305735615589214. 
510 Balkwill LL, Thompson WF. 1999. A Cross-Cultural Investigation of the Perception of Emotion

511

512

513

514

515

516

517

518

519

520

521

522

523

524

525

526

527

528

529

530

531

532

533

534

535

536

537

538

539

540

541

542

543

544

545

546

547

548 in Music: Psychophysical and Cultural Cues. Music Perception 17:43-64 DOI:10.2307/40285811.

Batcho KI, DaRin ML, Nave AM, Yaworsky RR. 2008. Nostalgia and identity in song lyrics. Psychology of Aesthetics, Creativity, and the Arts. 2:236-244 DOI:10.1037/19313896.2.4.236.

Baker F, Wigram T, Stott D, McFerran K. 2009. Therapeutic songwriting in music therapy, Part II: Comparing the literature with practice across diverse clinical populations. Nordic Journal of Music Therapy 18:32-56 DOI: 10.1080/08098130802496373.

Besson M, Faïta F, Peretz I, Bonnel AM, Requin J. 1998. Singing in the Brain: Independence of Lyrics and Tunes. Psychological Science 9:494-498 DOI: 10.1111/1467-9280.00091.

Bonnel AM, Faita F, Peretz I, Besson M. 2001. Divided attention between lyrics and tunes of operatic songs: Evidence for independent processing. Perception \& psychophysics. 63(7):1201-1213 DOI: 10.3758/BF03194534.

Brattico E, Alluri V, Bogert B, Jacobsen T, Vartiainen N, Nieminen S, Tervaniemi M. 2011. A Functional MRI Study of Happy and Sad Emotions in Music with and without Lyrics. Frontiers in Psychology 2. DOI: 10.3389/fpsyg.2011.00308.

Che W, Li Z, Liu T. 2010. LTP: a Chinese Language Technology Platform. In: Proceedings of the 23rd International Conference on Computational Linguistics: Demonstrations 13-16.

Chen S-H, Lee Y-S, Hsieh W-C, Wang J-C. 2015. Music emotion recognition using deep Gaussian process. In: 2015 Asia-Pacific Signal and Information Processing Association Annual Summit and Conference (APSIPA). Hong Kong: IEEE, 495-498. DOI: 10.1109/APSIPA.2015.7415321.

Chen Y-A, Yang Y-H, Wang J-C, Chen H. 2015. The AMG1608 dataset for music emotion recognition. In: 2015 IEEE International Conference on Acoustics, Speech and Signal Processing (ICASSP). South Brisbane, Queensland, Australia: IEEE, 693-697. DOI: 10.1109/ICASSP.2015.7178058.

Delbouys R, Hennequin R, Piccoli F, Royo-Letelier J, Moussallam M. 2018. Music mood detection based on audio and lyrics with deep neural net. In: Proceedings of the 19th International Society for Music Information Retrieval Conference (ISMIR), 370-375.

Deng JJ, Leung CHC, Milani A, Chen L. 2015. Emotional States Associated with Music: Classification, Prediction of Changes, and Consideration in Recommendation. ACM Transactions on Interactive Intelligent Systems 5:1-36. DOI: 10.1145/2723575.

Dingle GA, Kelly PJ, Flynn LM, Baker FA. 2015. The influence of music on emotions and cravings in clients in addiction treatment: A study of two clinical samples. The Arts in Psychotherapy 45:18-25. DOI: 10.1016/j.aip.2015.05.005.

Downie JS. 2008. The music information retrieval evaluation exchange (2005-2007): A window into music information retrieval research. Acoustical Science and Technology 29:247-255. DOI: $10.1250 /$ ast.29.247.

Peer] Comput. Sci. reviewing PDF | (CS-2021:07:63905:1:0:NEW 29 Sep 2021) 
549 Fedorenko E, Patel A, Casasanto D, Winawer J, Gibson E. 2009. Structural integration in 550 language and music: Evidence for a shared system. Memory \& Cognition 37:1-9. DOI:

$551 \quad 10.3758 / \mathrm{MC} .37 .1 .1$.

552 Freeman BC. 2012. No Eminems in Asia: What's in a song? Analyzing lyrics of Top 40 songs in

553

554

555

556

557

558

559

560

561

562

563

564

565

566

567

568

569

570

571

572

573

574

575

576

577

578

579

580

581

582

583

584

585

586 the East \& West. In: 3rd Inter-Asia Popular Music Studies (IAPMS). Available at https://www.researchgate.net/publication/272997075_No_Eminems_in_Asia_Analyzing_ly rics_of_Top_40_songs_in_the_East_West

Gabrielsson A. 2016. The relationship between musical structure and perceived expression. In: Susan H, Ian C, Michael Thaut T, eds. The Oxford Handbook of Music Psychology. Oxford: Oxford University Press, 215-232.

Gao R, Hao B, Li H, Gao Y, Zhu T. 2013. Developing Simplified Chinese Psychological Linguistic Analysis Dictionary for Microblog. In: Imamura K, Usui S, Shirao T, Kasamatsu T, Schwabe L, Zhong N eds. Brain and Health Informatics. Lecture Notes in Computer Science. Cham: Springer International Publishing, 359-368. DOI: 10.1007/978-3-31902753-1_36.

Gendron M, Lindquist KA, Barsalou L, Barrett LF. 2012. Emotion words shape emotion percepts. Emotion 12:314-325. DOI: 10.1037/a0026007.

Gordon RL, Schön D, Magne C, Astésano C, Besson M. 2010. Words and Melody Are Intertwined in Perception of Sung Words: EEG and Behavioral Evidence. PLoS ONE 5:e9889. DOI: 10.1371 /journal.pone.0009889.

Greenberg DM, Kosinski M, Stillwell DJ, Monteiro BL, Levitin DJ, Rentfrow PJ. 2016. The Song Is You: Preferences for Musical Attribute Dimensions Reflect Personality. Social Psychological and Personality Science 7:597-605. DOI: 10.1177/1948550616641473.

Greer T, Ma B, Sachs M, Habibi A, Narayanan S. 2019. A Multimodal View into Music's Effect on Human Neural, Physiological, and Emotional Experience. In: Proceedings of the 27th ACM International Conference on Multimedia. Nice France: ACM, 167-175. DOI: $\underline{10.1145 / 3343031.3350867 .}$.

Haselmayer M, Jenny M. 2017. Sentiment analysis of political communication: combining a dictionary approach with crowdcoding. Quality \& Quantity 51:2623-2646. DOI: 10.1007/s11135-016-0412-4.

Havlena W, abd Holak SL. 1991. "The good old days": Observations on nostalgia and its role in consumer behavior. Advances in Consumer Research 18:323-329.

Holbrook MB, Anand P. 1990. Effects of Tempo and Situational Arousal on the Listener's Perceptual and Affective Responses to Music. Psychology of Music 18:150-162. DOI: 10.1177/0305735690182004.

Hu X, Downie JS, Ehmann AF. 2009. Lyric text mining in music mood classification. In: Proceedings of the 10th International Society for Music Information Retrieval Conference (ISMIR), 411-416. 
$587 \mathrm{Hu}$ Y, Chen X, Yang D. 2009. Lyric-based Song Emotion Detection with Affective Lexicon and

588

589

590

591

592

593

594

595

596

597

598

599

600

601

602

603

604

605

606

607

608

609

610

611

612

613

614

615

616

617

618

619

620

621

622

623

624 Fuzzy Clustering Method. In: Proceedings of the 10th International Society for Music Information Retrieval Conference (ISMIR), 123-128.

Hunter PG, Schellenberg EG. 2010. Music and Emotion. In Mari RJ, Richard RF, Arthur NP, eds. Music Perception. Springer, 129-164.

Juslin PN, Laukka P. 2004. Expression, Perception, and Induction of Musical Emotions: A Review and a Questionnaire Study of Everyday Listening. Journal of New Music Research 33:217-238. DOI: 10.1080/0929821042000317813.

Juslin PN, Sloboda JA. 2001. Music and Emotion: Introduction. In: Juslin PN, Sloboda JA, eds. Music and Emotion: Theory and Research. Oxford: Oxford University Press, 3-19.

Kahng AB, Mantik S, Markov IL. 2002. Min-max placement for large-scale timing optimization. In: Proceedings of the 2002 international symposium on Physical design - ISPD '02. San Diego, CA, USA: ACM Press, 143. DOI: 10.1145/505388.505423.

Kaur R, Verma P. 2017. Sentiment analysis of movie reviews: A study of machine learning algorithms with various feature selection methods. International Journal of Computer Sciences and Engineering 5:113-121.

Kolinsky R, Lidji P, Peretz I, Besson M, Morais J. 2009. Processing interactions between phonology and melody: Vowels sing but consonants speak. Cognition 112:1-20. DOI: 10.1016/j.cognition.2009.02.014.

Lang PJ. 1995. The emotion probe: Studies of motivation and attention. American psychologist 50: 372-385. DOI: 10.1037/0003-066X.50.5.372

Laurier C, Grivolla J, Herrera P. 2008. Multimodal Music Mood Classification Using Audio and Lyrics. In: 2008 Seventh International Conference on Machine Learning and Applications. San Diego, CA, USA: IEEE, 688-693. DOI: 10.1109/ICMLA.2008.96.

Liu B. 2012. Sentiment Analysis and Opinion Mining. Synthesis Lectures on Human Language Technologies 5:1-167. DOI: 10.2200/S00416ED1V01Y201204HLT016.

Liu SM, Chen J-H. 2015. A multi-label classification based approach for sentiment classification. Expert Systems with Applications 42:1083-1093. DOI: 10.1016/j.eswa.2014.08.036.

Lu K, Wu J. 2019. Sentiment Analysis of Film Review Texts Based on Sentiment Dictionary and SVM. In: Proceedings of the 2019 3rd International Conference on Innovation in Artificial Intelligence - ICIAI 2019. Suzhou, China: ACM Press, 73-77. DOI: $10.1145 / 3319921.3319966$.

Malheiro R, Oliveira HG, Gomes P, Paiva RP. 2016. Keyword-based Approach for Lyrics Emotion Variation Detection: In: Proceedings of the 8th International Joint Conference on Knowledge Discovery, Knowledge Engineering and Knowledge Management. Porto, Portugal: SCITEPRESS - Science and Technology Publications, 33-44. DOI: $\underline{10.5220 / 0006037300330044 .}$.

Peer] Comput. Sci. reviewing PDF | (CS-2021:07:63905:1:0:NEW 29 Sep 2021) 
625 Malheiro R, Panda R, Gomes P, Paiva RP. 2018. Emotionally-Relevant Features for

626

627

628

629

630

631

632

633

634

635

636

637

638

639

640

641

642

643

644

645

646

647

648

649

650

651

652

653

654

655

656

657

658

659

660

661

662

663 9:240-254. DOI: 10.1109/TAFFC.2016.2598569.

Malheiro R, Panda R, Gomes P, Paiva R. 2016. Bi-modal music emotion recognition: Novel lyrical features and dataset. In: 9th International Workshop on Music and Machine Learning-MML'2016-in conjunction with the European Conference on Machine Learning and Principles and Practice of Knowledge Discovery in Databases-ECML/PKDD 2016.

McFee B, Raffel C, Liang D, Ellis DP, McVicar M, Battenberg E, Nieto O. 2015. librosa: Audio and music signal analysis in python. In: Proceedings of the 14th python in science conference, 8:18-25.

McVicar M, Freeman T, De Bie T. 2011. Mining the Correlation between Lyrical and Audio Features and the Emergence of Mood. In: Proceedings of the 12th International Society for Music Information Retrieval Conference (ISMIR), 783-788.

Meyer BT, Kollmeier B. 2009. Complementarity of MFCC, PLP and Gabor features in the presence of speech-intrinsic variabilities. In: Tenth Annual Conference of the International Speech Communication Association, 2755-2758.

Morton JB, Trehub SE. 2001. Children's Understanding of Emotion in Speech. Child Development 72:834-843. DOI: 10.1111/1467-8624.00318.

Morton JB, Trehub SE. 2007. Children's judgements of emotion in song. Psychology of Music 35:629-639. DOI: 10.1177/0305735607076445.

O'Callaghan C, Grocke D. 2009. Lyric analysis research in music therapy: Rationales, methods and representations. The Arts in Psychotherapy 36:320-328. DOI: 10.1016/j.aip.2009.09.004.

Panda R, Malheiro R, Rocha B, Oliveira A, Paiva RP. 2013. Multi-modal music emotion recognition: A new dataset, methodology and comparative analysis. In: International Symposium on Computer Music Multidisciplinary Research, 1-13.

Peng H, Cambria E, Hussain A. 2017. A Review of Sentiment Analysis Research in Chinese Language. Cognitive Computation 9:423-435. DOI: 10.1007/s12559-017-9470-8.

Pennebaker JW. 2004. Theories, Therapies, and Taxpayers: On the Complexities of the Expressive Writing Paradigm. Clinical Psychology: Science and Practice 11:138-142. DOI: $10.1093 /$ clipsy.bph063.

Pennebaker JW, Francis ME, Booth RJ. 2001. Linguistic inquiry and word count (LIWC): LIWC2001. Available at http://downloads.liwc.net.s3.amazonaws.com/

Petrie KJ, Pennebaker JW, Sivertsen B. 2008. Things we said today: A linguistic analysis of the Beatles. Psychology of Aesthetics, Creativity, and the Arts 2:197-202. DOI: $10.1037 / \mathrm{a} 0013117$.

Pettijohn TF, Sacco DF. 2009. The Language of Lyrics: An Analysis of Popular Billboard Songs Across Conditions of Social and Economic Threat. Journal of Language and Social Psychology 28:297-311. DOI: 10.1177/0261927X09335259. 
664 Pieschl S, Fegers S. 2016. Violent Lyrics = Aggressive Listeners?: Effects of Song Lyrics and

665

666

667

668

669

670

671

672

673

674

675

676

677

678

679

680

681

682

683

684

685

686

687

688

689

690

691

692

693

694

695

696

697

698

699

700

701

702 Tempo on Cognition, Affect, and Self-Reported Arousal. Journal of Media Psychology 28:32-41. DOI: 10.1027/1864-1105/a000144.

Poulin-Charronnat B, Bigand E, Madurell F, Peereman R. 2005. Musical structure modulates semantic priming in vocal music. Cognition 94:B67-B78. DOI: 10.1016/j.cognition.2004.05.003.

Proverbio AM, Benedetto F, Guazzone M. 2020. Shared neural mechanisms for processing emotions in music and vocalizations. European Journal of Neuroscience 51:1987-2007. DOI: $10.1111 /$ ejn.14650.

Rachman FH, Samo R, Fatichah C. 2019. Song Emotion Detection Based on Arousal-Valence from Audio and Lyrics Using Rule Based Method. In: 2019 3rd International Conference on Informatics and Computational Sciences (ICICoS). Semarang, Indonesia: IEEE, 1-5. DOI: $10.1109 /$ ICICoS48119.2019.8982519.

Schmidt EM, Turnbull D, Kim YE. 2010. Feature selection for content-based, time-varying musical emotion regression. In: Proceedings of the international conference on Multimedia information retrieval - MIR '10. Philadelphia, Pennsylvania, USA: ACM Press, 267. DOI: 10.1145/1743384.1743431.

Schwartz JW, Gouzoules H. 2019. Decoding human screams: perception of emotional arousal from pitch and duration. Behaviour 156:1283-1307. DOI: $10.1163 / 1568539 \mathrm{X}-00003566$.

Sen A, Srivastava M. 2012. Multiple regression. In: Sen A, Srivastava M, eds. Regression analysis: theory, methods, and applications. Springer Science \& Business Media: 28-49.

Serafine M. 1984. Integration of melody and text in memory for songs. Cognition 16:285-303. DOI: $10.1016 / 0010-0277(84) 90031-3$.

Serafine ML, Davidson J, Crowder RG, Repp BH. 1986. On the nature of melody-text integration in memory for songs. Journal of Memory and Language 25:123-135. DOI: 10.1016/0749-596X(86)90025-2.

Silverman MJ. 2020. Therapeutic songwriting to address distress tolerance for adults on an acute care mental health unit: A pilot study. The Arts in Psychotherapy 71:101716. DOI: 10.1016/j.aip.2020.101716.

Slatcher RB, Pennebaker JW. 2006. How Do I Love Thee? Let Me Count the Words: The Social Effects of Expressive Writing. Psychological Science 17:660-664. DOI: 10.1111/j.14679280.2006.01762.x.

Sousou SD. 1997. Effects of Melody and Lyrics on Mood and Memory. Perceptual and Motor Skills 85:31-40. DOI: 10.2466/pms.1997.85.1.31.

Speck JA, Schmidt EM, Morton BG, Kim YE. 2011. A Comparative Study of Collaborative vs. Traditional Musical Mood Annotation. In: Proceedings of the 12th International Society for Music Information Retrieval Conference (ISMIR), 549-554.

Stratton VN, Zalanowski AH. 1994. Affective Impact of Music Vs. Lyrics. Empirical Studies of the Arts 12:173-184. DOI: 10.2190/35T0-U4DT-N09Q-LQHW. 
703

704

705

706

707

708

709

710

711

712

713

714

715

716

717

718

719

720

721

722

723

724

725

726

727

728

729

730

731

732

733

734

735

736

737

738

739

740

Swaminathan S, Schellenberg EG. 2015. Current Emotion Research in Music Psychology. Emotion Review 7:189-197. DOI: 10.1177/1754073914558282.

van Besouw RM, Howard DM, Ternström S. 2005. Towards an understanding of speech and song perception. Logopedics Phoniatrics Vocology 30:129-135. DOI: 10.1080/14015430500262160.

van Waesberghe JS. 1955. A Textbook of Melody: A course in functional melodic analysis. American Institute of Musicology Press.

Vempala NN, Russo FA. 2018. Modeling Music Emotion Judgments Using Machine Learning Methods. Frontiers in Psychology 8:2239. DOI: 10.3389/fpsyg.2017.02239.

Vidas D, Calligeros R, Nelson NL, Dingle GA. 2020. Development of emotion recognition in popular music and vocal bursts. Cognition and Emotion 34:906-919. DOI: 10.1080/02699931.2019.1700482.

Viega M, Baker FA. 2017. What's in a song? Combining analytical and arts-based analysis for songs created by songwriters with neurodisabilities. Nordic Journal of Music Therapy 26:235-255. DOI: 10.1080/08098131.2016.1205651.

Wang J-C, Yang Y-H, Wang H-M, Jeng S-K. 2012. The acoustic emotion gaussians model for emotion-based music annotation and retrieval. In: Proceedings of the 20th ACM international conference on Multimedia - MM '12. Nara, Japan: ACM Press, 89. DOI: $10.1145 / 2393347.2393367$.

Xu L, Li L, Jiang Z, Sun Z, Wen X, Shi J, Sun R, Qian X. 2021. A Novel Emotion Lexicon for Chinese Emotional Expression Analysis on Weibo: Using Grounded Theory and SemiAutomatic Methods. IEEE Access 9:92757-92768. DOI: 10.1109/ACCESS.2020.3009292.

Xu L, Wen X, Shi J, Li S, Xiao Y, Wan Q, Qian X. 2020. Effects of individual factors on perceived emotion and felt emotion of music: Based on machine learning methods. Psychology of Music:030573562092842. DOI: 10.1177/0305735620928422.

Xu L, Yun Z, Sun Z, Wen X, Wang J, Qian X. 2020. PSIC3839: Predicting the Overall Emotion and Depth of Entire Songs. Available at https://github.com/x12218066/PSIC3839.

Xu L, Zheng Y, Xu D, Xu L. 2021. Predicting the Preference for Sad Music: The Role of Gender, Personality, and Audio Features. IEEE Access 9:92952-92963. DOI: 10.1109/ACCESS.2021.3090940.

Yang X, Dong Y, Li J. 2018. Review of data features-based music emotion recognition methods. Multimedia Systems 24:365-389. DOI: 10.1007/s00530-017-0559-4.

Yu Y, Tang S, Raposo F, Chen L. 2019. Deep Cross-Modal Correlation Learning for Audio and Lyrics in Music Retrieval. ACM Transactions on Multimedia Computing, Communications, and Applications 15:1-16. DOI: 10.1145/3281746.

Yu Y, Wu D, Zhang J, Fang P. 2019. Lyrics only or lyrics with music? The effect of different lyric conditions on prosocial-related outcomes. PsyCh Journal 8:503-512. DOI: 10.1002/pchj.269. 
741 Zacharopoulou K, Kyriakidou A. 2009. A cross-cultural comparative study of the role of musical

742 structural features in the perception of emotion in Greek traditional music. Journal of

743 Interdisciplinary Music Studies 3:1-15.

744 Zhang C, Tong T, Bu Y. 2019. Examining differences among book reviews from various online

745 platforms. Online Information Review 43:1169-1187. DOI: 10.1108/OIR-01-2019-0037.

746 Zhao N, Jiao D, Bai S, Zhu T. 2016. Evaluating the Validity of Simplified Chinese Version of

$747 \quad$ LIWC in Detecting Psychological Expressions in Short Texts on Social Network Services.

$748 \quad$ PLOS ONE 11:e0157947. DOI: 10.1371/journal.pone.0157947.

749 Zhou J, Chen X, Yang D. 2019. Multimodel Music Emotion Recognition Using Unsupervised

750 Deep Neural Networks. In: Li W, Li S, Shao X, Li Z eds. Proceedings of the 6th

$751 \quad$ Conference on Sound and Music Technology (CSMT). Lecture Notes in Electrical

752 Engineering. Singapore: Springer Singapore, 27-39. DOI: $\underline{\text { 10.1007/978-981-13-8707-4 } 3 .}$

753 


\section{Table $\mathbf{1}$ (on next page)}

Correlation between lyric features and perceived arousal in music

PastM: proportion of past tense markers; Insight: proportion of words related to insight; Time: proportion of words related to time; Achieve: proportion of words related to achievement; tPast: proportion of words related to the past; WordCount: the total number of words; WordPerSentence: average number of words per sentence; RateLatinWord: the ratio of Latin words. ${ }^{* *}$ Correlation is significant at the 0.01 level (2-tailed). 
1 Table 1 Correlation between lyric features and perceived arousal in music

\begin{tabular}{|c|c|c|c|c|c|c|c|c|c|}
\hline & 1 & 2 & 3 & 4 & 5 & 6 & 7 & 8 & 9 \\
\hline 1. Arousal & 1 & & & & & & & & \\
\hline 2. PastM & $-.121^{* \star}$ & 1 & & & & & & & \\
\hline 3. Insight & $-.122^{* *}$ & $.168^{* *}$ & 1 & & & & & & \\
\hline 4. Time & $-.115^{\star *}$ & $.299^{* *}$ & $.139^{* *}$ & 1 & & & & & \\
\hline 5. Achieve & $.111^{\star *}$ & 0.023 & $.146^{\star *}$ & -0.006 & 1 & & & & \\
\hline 6. tPast & $-.124^{\star *}$ & $.517^{* *}$ & $.153^{* *}$ & $.322^{* *}$ & 0.014 & 1 & & & \\
\hline 7. WordCount & $.206^{* *}$ & $.056^{* *}$ & $.080^{* *}$ & 0.004 & $.073^{\star *}$ & 0.014 & 1 & & \\
\hline 8. WordPerSentence & $.179^{\star *}$ & $.062^{* *}$ & $.105^{\star \star}$ & 0.021 & $.088^{* *}$ & 0.025 & $.873^{* *}$ & 1 & \\
\hline 9. RateLatinWord & $.183^{* *}$ & -0.029 & 0.02 & -0.032 & $.103^{* *}$ & -0.031 & $.174^{* *}$ & $.114^{* *}$ & 1 \\
\hline
\end{tabular}

2 Notes: ${ }^{* *}$ Correlation is significant at the 0.01 level (2-tailed).

3 Abbreviations: PastM: proportion of past tense markers; Insight: proportion of words related to insight; Time: 4 proportion of words related to time; Achieve: proportion of words related to achievement; tPast: proportion of words 5 related to the past; WordCount: the total number of words; WordPerSentence: average number of words per sentence; 6 RateLatinWord: the ratio of Latin words. 


\section{Table 2 (on next page)}

Correlation between lyric features and perceived valence in music

Adverb: proportion of adverbs; TenseM: proportion of tense markers; PastM: proportion of past tense markers; NegEmo: proportion of negative emotion words; Sad: proportion of words related to sadness; CogMech: proportion of words related to cognition; Tentat: proportion of words related to tentativeness; tPast: proportion of words related to the past. **Correlation is significant at the 0.01 level (2-tailed). 
1 Table 2 Correlation between lyric features and perceived valence in music

\begin{tabular}{|c|c|c|c|c|c|c|c|c|c|}
\hline & 1 & 2 & 3 & 4 & 5 & 6 & 7 & 8 & 9 \\
\hline 1. Arousal & 1 & & & & & & & & \\
\hline 2. PastM & $-.121^{* *}$ & 1 & & & & & & & \\
\hline 3. Insight & $-.122^{\star *}$ & $.168^{\star *}$ & 1 & & & & & & \\
\hline 4. Time & $-.115^{\star *}$ & $.299^{\star \star}$ & $.139^{* *}$ & 1 & & & & & \\
\hline 5. Achieve & $.111^{* *}$ & 0.023 & $.146^{* *}$ & -0.006 & 1 & & & & \\
\hline 6. tPast & $-.124^{\star *}$ & $.517^{\star \star}$ & $.153^{\star *}$ & $.322^{* *}$ & 0.014 & 1 & & & \\
\hline 7. WordCount & $.206^{* *}$ & $.056^{\star *}$ & $.080^{* *}$ & 0.004 & $.073^{* *}$ & 0.014 & 1 & & \\
\hline 8. WordPerSentence & $.179^{* *}$ & $.062^{* *}$ & $.105^{\star *}$ & 0.021 & $.088^{* *}$ & 0.025 & $.873^{* *}$ & 1 & \\
\hline 9. RateLatinWord & $.183^{\star *}$ & -0.029 & 0.02 & -0.032 & $.103^{\star *}$ & -0.031 & $.174^{\star *}$ & $.114^{\star \star}$ & 1 \\
\hline
\end{tabular}

2 Notes: ${ }^{* *}$ Correlation is significant at the 0.01 level (2-tailed).

3 Abbreviations: Adverb: proportion of adverbs; TenseM: proportion of tense markers; PastM: proportion of past tense 4 markers; NegEmo: proportion of negative emotion words; Sad: proportion of words related to sadness; CogMech: 5 proportion of words related to cognition; Tentat: proportion of words related to tentativeness; $t$ Past: proportion of words 6 related to the past. 


\section{Table 3(on next page)}

The best performing parameters for each random forest regression

AF indicates audio features; LF indicates lyric features; and CF indicates combined features. 
1 Table 3 The best performing parameters for each random forest regression

\begin{tabular}{|c|c|c|c|c|c|c|}
\hline Ground & \multirow{2}{*}{ Inputs } & \multicolumn{5}{|c|}{ Parameters } \\
\hline truth & & n_estimators & max_depth & min_samples_leaf & min_samples_split & max_features \\
\hline \multirow{3}{*}{ Arousal } & $\mathrm{AF}$ & 156 & 10 & 8 & 18 & 0.2 \\
\hline & LF & 196 & 50 & 5 & 8 & 0.8 \\
\hline & $\mathrm{CF}$ & 136 & 27 & 3 & 12 & 0.4 \\
\hline \multirow{3}{*}{ Valenc } & AF & 179 & 15 & 13 & 22 & 0.5 \\
\hline & & & & & & \\
\hline & LF & 193 & 38 & 3 & 8 & 0.8 \\
\hline \multicolumn{7}{|l|}{ e } \\
\hline & CF & 191 & 43 & 5 & 25 & 0.6 \\
\hline
\end{tabular}

2 Abbreviations: AF indicates audio features; LF indicates lyric features; and CF indicates combined features. 
Figure 1

The proposed modeling method.

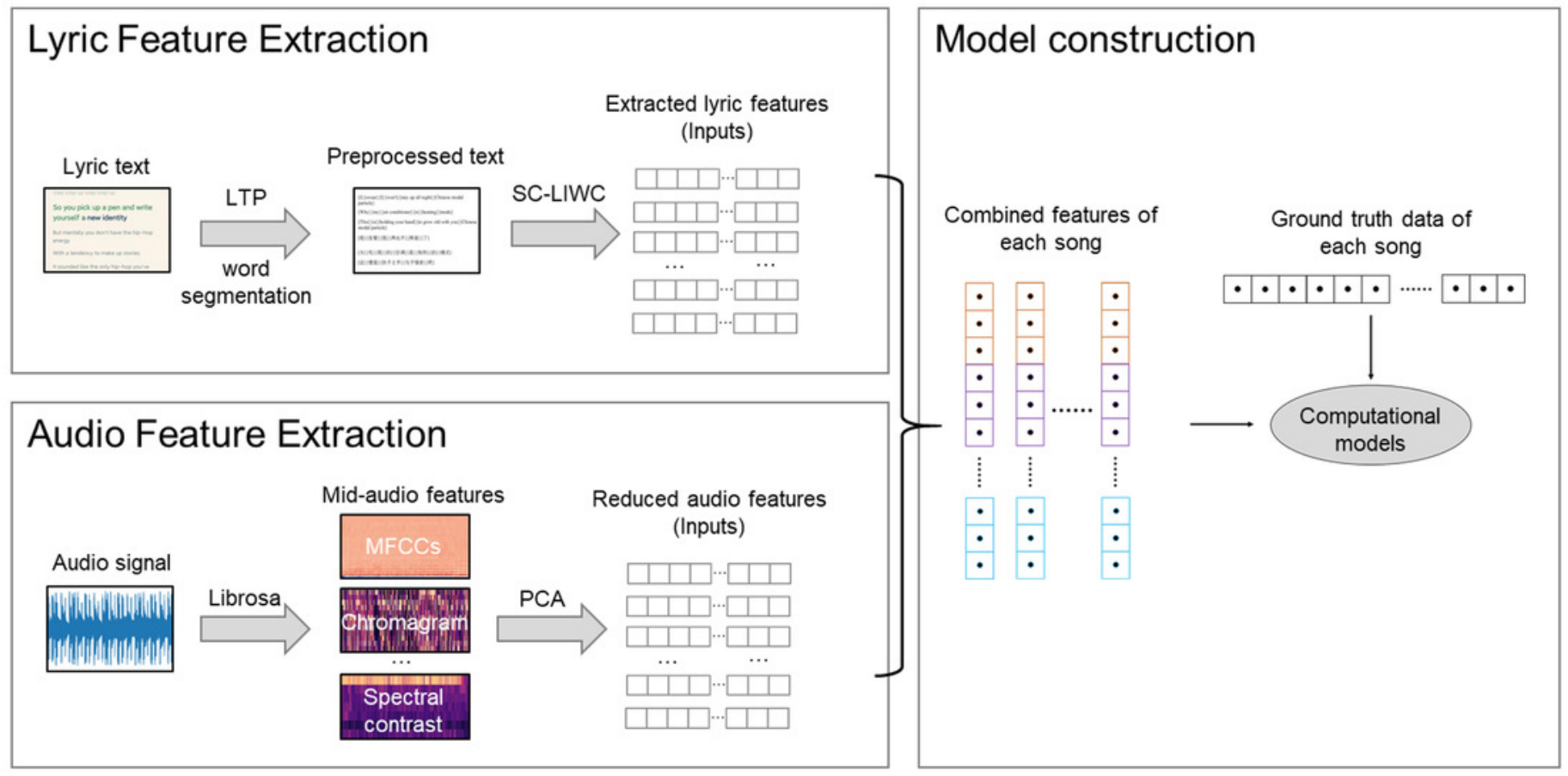


Figure 2

Data distribution of the 2372 Chinese songs in this study.

(a) Distribution of annotated emotions in the valence-arousal emotion space. (b) Word clouds of the top words used in each quadrant of the valence-arousal emotion space. The font size depends on the usage frequency of the word (positive correlation).
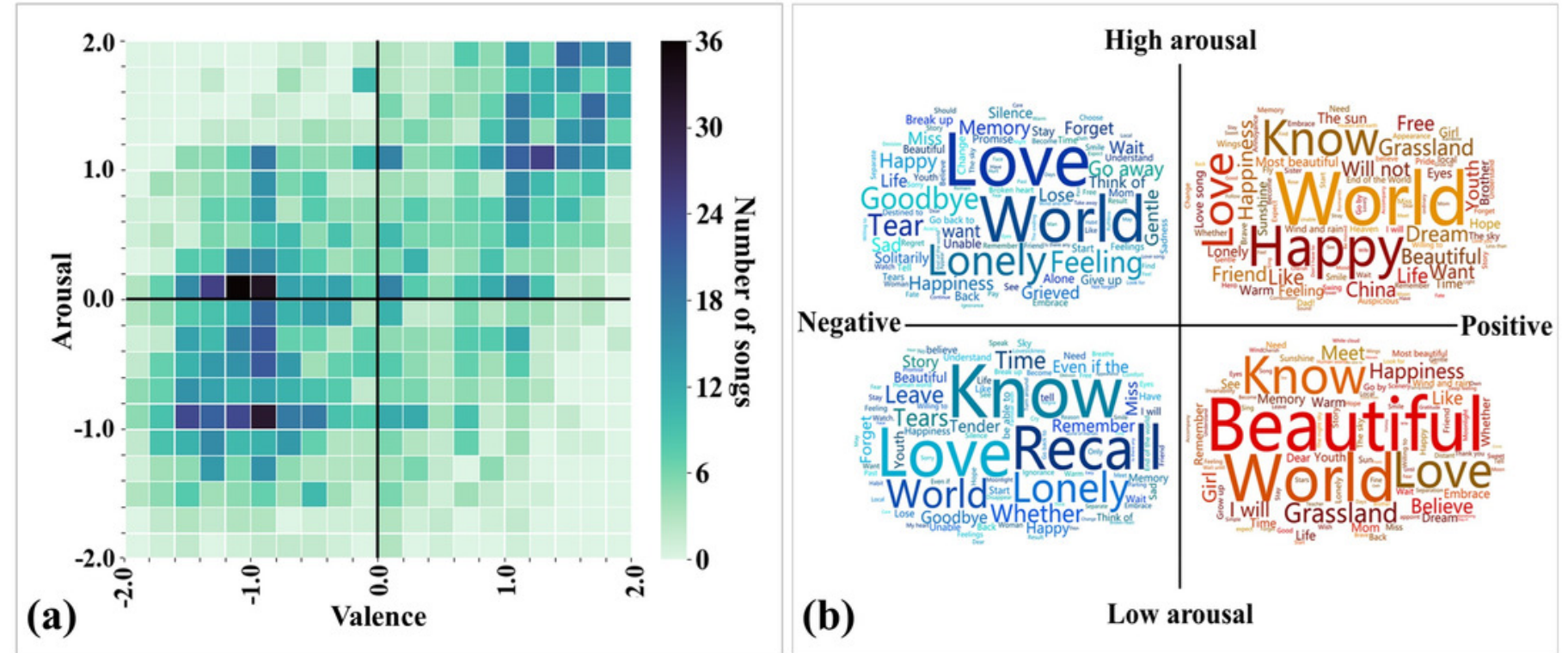
Figure 3

Performance of constructed MER models with different inputs and algorithms.

(a) Prediction results of perceived arousal recognition models, measured by $R^{2}$ statistics. (b) Prediction results of perceived arousal recognition models, measured by RMSE. (c) Prediction results of perceived valence recognition models, measured by $R^{2}$ statistics. (d) Prediction results of perceived valence recognition models, measured by RMSE. Error bars indicate the standard deviations.

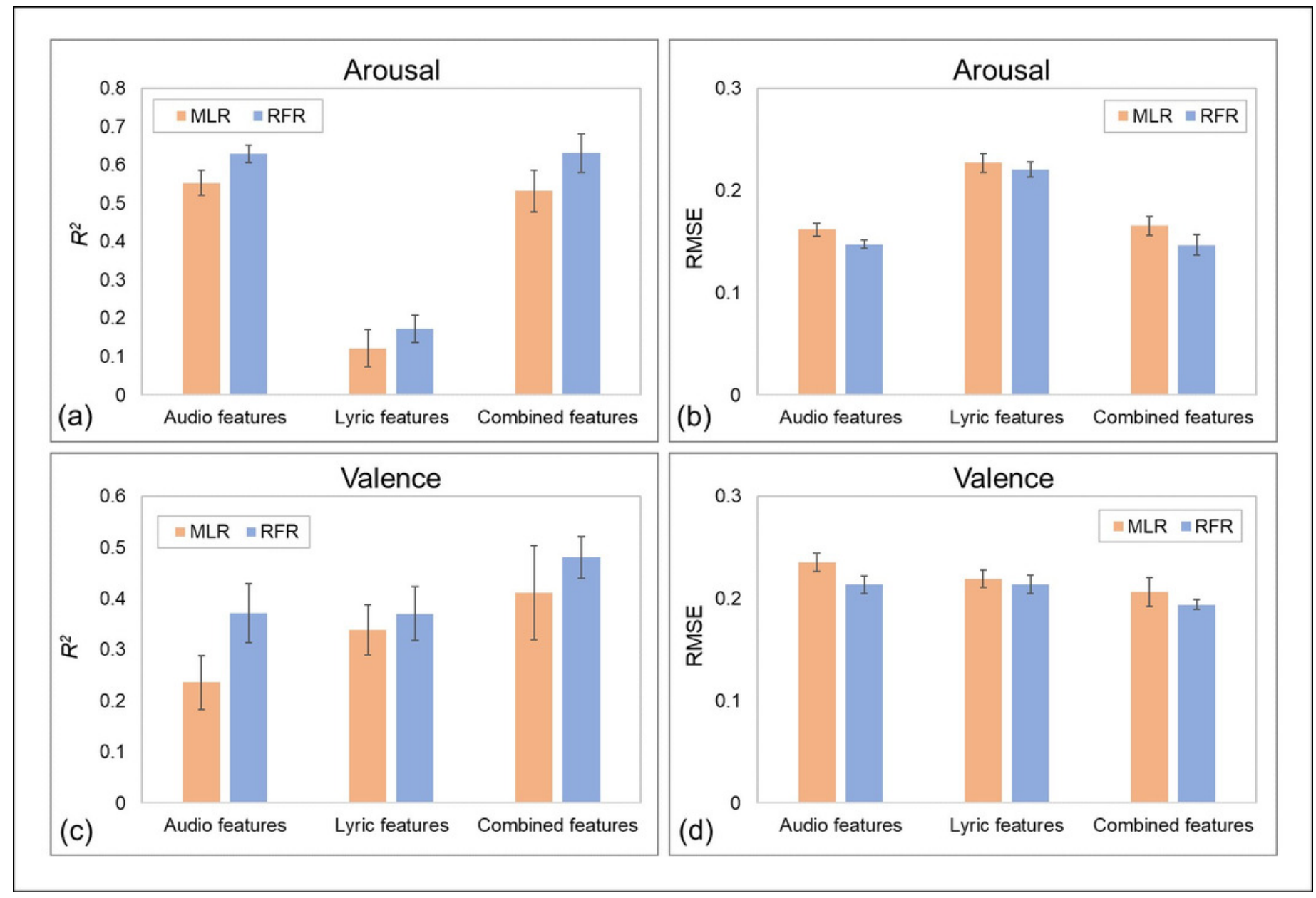


Figure 4

Distribution of feature importance for RFR-based recognition models of perceived arousal and perceived valence.

Arranged in descending order of the mean value, the top 30 features were included for visibility, and the trend of the remaining features was approximately the same. Error bars indicate the standard deviations.
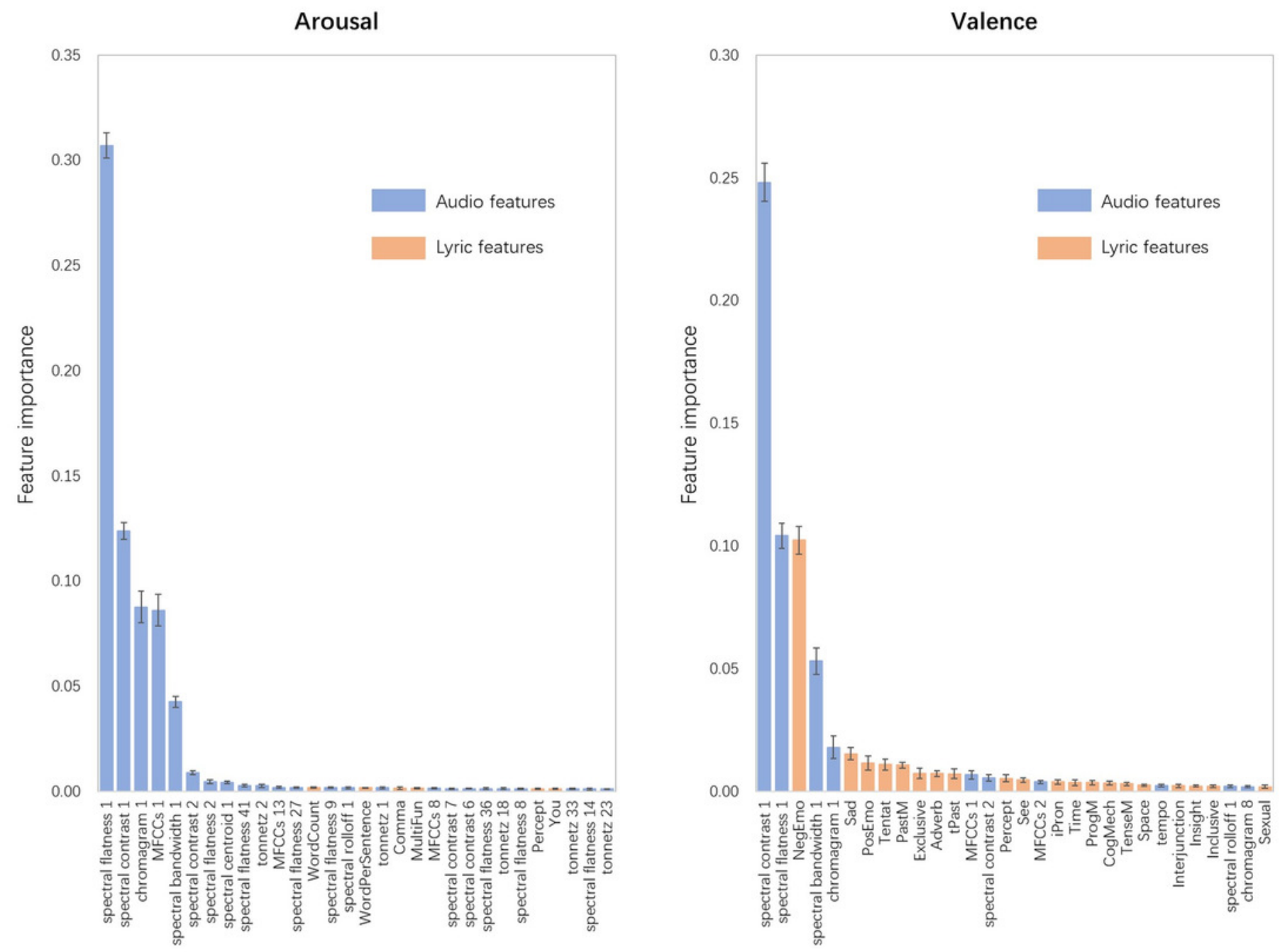